\section{2 \\ Dissociation Constants and Dissociation Velocities of Substituted Pyruvic Acids}

IT has been shown ${ }^{1}$ that weak acids are very often reduced polarographically in two steps. The more positive step belongs to the undissociated acid, the more negative to the acid ion. The total height of these two steps remains constant; but their ratio is changed in a characteristic way with the $p \mathrm{H}$ of the solution. If we plot this ratio against the $p \mathrm{H}$, we obtain a curve which resembles very closely a dissociation curve. The $p K$ of this dissociation curve is, however, generally shifted several $p \mathrm{H}$ units to higher values in comparison with the true acidity of the particular acid.

This shift is due ${ }^{1}$ to the rapid recombination of the acid ions with protons in the neighbourhood of the dropping electrode. The mathematical theory developed ${ }^{1,2,3}$ introduced simplifications, but accounted for all the facts. Later a rigorous formulation was given, and it is possible ${ }^{4}$ to calculate the velocity constant of the recombination reaction accurately from the shift of the $p K$ value.

In this calculation it was assumed that the recombination is due solely to the reaction between acid ions $A c^{\prime}$ and free hydrogen ions. Therefore, for the calculation of the effective velocity constant $k_{\mathrm{ef}}$, the simplified form of the reaction velocity expression $k_{\text {ef }}\left[A c^{\prime}\right]\left[\mathrm{H}^{+}\right]$is used instead of the more accurate

$k_{\mathrm{H}}+\left[A c^{\prime}\right]\left[\mathrm{H}^{+}\right]+k_{\mathrm{H}_{2} \mathrm{O}}\left[A c^{\prime}\right]\left[\mathrm{H}_{2} \mathrm{O}\right]+k_{a c \mathrm{H}}\left[A c^{\prime}\right][a c \mathrm{H}$

that is,

$$
k_{\mathrm{ef}}\left[\mathrm{H}^{+}\right]=k_{\mathrm{H}+}\left[\mathrm{H}^{+}\right]+k_{\mathrm{H}, \mathrm{O}}\left[\mathrm{H}_{2} \mathrm{O}\right]+k_{a c \mathrm{H}}[a c \mathrm{H}] \text {, }
$$

where $a c \mathrm{H}$ represents the acid of the buffer solution.

We can, however, compare $k_{\text {ef }}$ for different acids if we use the same buffer solution. Also changes in buffer concentration have only a very small influence. In view of the importance of the relationship between the dissociation constants and dissociation velocities of acids for the theory of acid-base catalysis and for the interpretation of the Brønsted equation, we have determined the dissociation constants and $k_{\text {ef }}$ of a number of substituted pyruvic acids. The results are given in the accompanying table. The buffer solution used was the Britton-Robinson ${ }^{5}$ universal buffer.

\begin{tabular}{|l|c|c|c|c|}
\hline \multicolumn{1}{|c|}{ Acid } & $p K$ & $p K_{\boldsymbol{p}}$ & $t$ & $\log k \mathrm{ef}$ \\
\hline 3,4 Dimethoxyphenyl- & & & & \\
pyruvic & $3 \cdot 10$ & $7 \cdot 6$ & $2 \cdot 90$ & $11 \cdot 24$ \\
Phenylpyruvic & $2 \cdot 68$ & $6 \cdot 72$ & $2 \cdot 93$ & $9 \cdot 89$ \\
Trimethylpyruvic & $2 \cdot 30$ & $4 \cdot 70$ & $2 \cdot 90$ & $6 \cdot 24$ \\
Pyruvic & $2 \cdot 50$ & $5 \cdot 85$ & $2 \cdot 97$ & $8 \cdot 33$ \\
Diphenylpyruvic & $2 \cdot 78$ & $6 \cdot 98$ & $3 \cdot 00$ & $10 \cdot 30$ \\
\hline
\end{tabular}

$t$, drop time of the mercury electrode; $p K$, real $p K$ determined by potentiometric titration; $p K_{p}$, apparent polarographic dissociation constant which gives the $p \mathbf{H}$ at which the positive and negative polarographic waves have the same height; log kef was obtained by the Koutecky and Brdicka calculation, namely, $\log k$ ef $=2\left(p K p+\frac{1}{2} \log \right.$ $\left.y_{1}\right)-p K-\log t ; y_{1}$ is a tabulated function (ref. 4).

Plotting $\log k_{\mathrm{ef}}$ against $p K$, it is found that the values lie on a smooth curve which is nearly a straight line (see graph). Several acids not belonging to the pyruvic acid series have been found to give $p K$ and $k_{\text {ef }}$ values which do not lie on this curve.
February 4, 1950 vol. 165

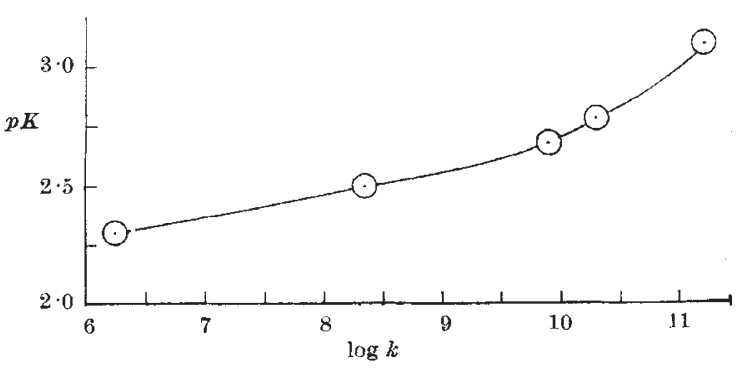

We hope to investigate other series of homologues and to eliminate completely the influence of the buffer by systematic variation of buffer concentration and extrapolation to zero.

Chemistry Department,

E. G. Clair

K. WIESNER

University of New Brunswick, Fredericton, N.B.

Aug. 18.

'Brdicka, R., and Wiesner, K., Coll., 12, 138 (1947); Chem. listy. 40, 66 (i 946$)$.

${ }^{2}$ Wiesner, K., Chem. listy, 41, 6 (1947).

3 Brdicka, R., Coll., 12, 212 (1947).

- Koutecky and Brdicka, Coll., 12, 338 (1947).

s Britton, H. T. S., "Hydrogen Ions" 225 (1932)

\section{Oxides of Praseodymium}

The two oxides of praseodymium described in the literature are the light green sesquioxide and the dark brown 'air-ignited' oxide. It is generally agreed that the composition of the latter approximates to $\operatorname{Pr}_{6} \mathrm{O}_{11}$. PrandtI and his school ${ }^{1}$ claim that praseodymium can exhibit a higher valeney of 5 , and on this basis designate the air-ignited oxide as $2 \mathrm{Pr}_{2} \mathrm{O}_{3} . \mathrm{Pr}_{2} \mathrm{O}_{5}$. Marsh' affirms, however, that praseodymium possesses a maximum valency of $\mathbf{4}$, a conclusion which is well supported by the work of Zintl and Morawietz ${ }^{3}$, and represents $\operatorname{Pr}_{6} \mathrm{O}_{11}$ as the double oxide, $4 \mathrm{PrO}_{2} . \mathrm{Pr}_{2} \mathrm{O}_{3}$.

Since $\mathrm{Pr}_{2} \mathrm{O}_{3}$ is derived from the lower of the two valence states of praseodymium, it might be expected that the potential quadrivalency (on energetic grounds quadrivalency appears more likely than pentavalency) of this metal will favour the formation of a non. stoichiometric phase with a range of composition on the axygen-rich side of the ideal formula. According to Wilson's ${ }^{4}$ theory of semi-conductors, these oxides would be deficit conductors showing positive-hole conduction. Conversely, $\mathrm{PrO}_{2}$, if partly denuded of oxygen, would give rise to oxides which are on the metal-rich side of the ideal formula and are electron conductors.

The present communication reports experiments which indicate that this is indeed the case, and that praseodymium forms a series of non-stoichiometric oxides in the range $\mathrm{PrO}_{1 \cdot 5}-\mathrm{PrO}_{2 \cdot 0}$. On this basis the air-ignited (that is, ignited in a partial pressure of $150 \mathrm{~mm}$. oxygen) oxide is non-stoichiometric in character and is properly represented by the formula $\mathrm{PrO}_{1 \cdot 83}$. No particular significance should be ascribed to the representation $\operatorname{Pr}_{6} \mathrm{O}_{11}$.

Tensiometric measurements on this oxide system, in conjunction with $\mathrm{X}$-ray diffraction patterns of oxides $\mathrm{PrO}_{1.500}, \mathrm{PrO}_{1.585}, \mathrm{PrO}_{1.658}, \mathrm{PrO}_{1.710}$ and $\mathrm{PrO}_{1.833}$, indicate that two oxide phases exist, one cubic and one hexagonal. The former is derived from 